\title{
Mobile Monitoring for Distributed Infrastructures
}

\author{
Bing Jiang, Alanson P. Sample, and Alexander V. Mamishev \\ Sensors, Energy, and Automation Laboratory, Department of Electrical Engineering, \\ University of Washington, Seattle, WA, USA 98195 \\ \{bjiang, alanson, mamishev\}@ee.washington.edu
}

\begin{abstract}
Efficient maintenance of distributed infrastructures requires accurate and up-to-date information to ensure reliable service. Existing monitoring techniques commonly rely on either data collected at scattered points or on costly manual inspection of suspect components by service personnel. Implementation of mobile robots for continuous autonomous monitoring of these infrastructures can provide a cost-effective means of gaining accurate information for maintenance planning. This paper offers a discussion on key system-level issues that must be addressed in any mobile monitoring project. These issues include: (a) economic analysis of incorporating mobile robotics into existing maintenance strategies, (b) the ability of the autonomous robot to negotiate diverse and unstructured environment, and (c) the feasibility of integrating needed sensors into a robotic platform. The example application examined in this paper is an autonomous robotic platform that monitors underground power cables for incipient failures.
\end{abstract}

Index Terms - Autonomous monitoring, mobile robot, crawler, sensors, distributed infrastructure, aging status, RFID.

\section{INTRODUCTION}

Distributed infrastructures are ubiquitous in today's industrial, civil, and commercial environments. As the complexity of these structures increases, new and innovative monitoring and maintenance techniques are becoming necessary to maintain and improve these systems. Examples of distributed infrastructures are oil and gas pipelines, transmission and distribution power grids, and sewage and waste disposal systems. These industrial systems occupy large territories and comprise many miles of linear structures. This paper focuses on an electric power distribution network as a case study system.

Existing techniques for monitoring power distribution cables require manual inspection by maintenance staff or by outside consultants. The instrumentation used for such tasks varies from simple hand-held devices to vans equipped with highly sensitive measurement equipment $[1,2]$. As a result, the amount of information known about a system is often limited to measurements at input and output terminals of a network branch. Consequently, it is not feasible to provide cost-effective

This project is supported primarily by the NSF CAREER Award \#0093716. It is also partly supported by the Electrical Energy Industrial Consortium and Advanced Power Technologies (APT) Center at the University of Washington. The industrial members of the APT Center include AREVA, Bonneville Power Administration, CESI, LG Industrial Systems, Mitsubishi Electric, PJM, and RET. and comprehensive monitoring of system parameters.

Autonomous robotics can potentially offer an effective and economical method for monitoring of distributed infrastructures. The mobile units can continuously take periodic measurements throughout the system. However, this vision for mobile robotics has not been realized so far for most types of infrastructures. This paper offers an analysis of the three key issues that must be addressed before autonomous robots can become an integral part of an infrastructure monitoring system. These issues are economic justification, environment negotiation capability, and sensor technology.

\section{A. Economic justification}

Without a solid economic justification, the introduction of mobile monitoring robots into a distributed infrastructure will remain a laboratory exercise. The new maintenance method must complement, but not disrupt the existing paradigms of system operation and maintenance. Traditional maintenance strategies fall primarily into one of the two categories: corrective maintenance and scheduled maintenance.

Corrective maintenance takes place as a response to a failure in the system. For example, a water distribution line may fail, causing a loss of service to the customer. The problem is corrected with the replacement of the failing section of pipe.

Scheduled maintenance is used when the unplanned loss of service is deemed to be highly undesirable. The system must be serviced on a predetermined schedule to ensure that failures do not occur. The downside of this type of maintenance is that premature replacement of infrastructure components leads to excessive economic losses.

Neither of these two options provides the best economic and service solutions. Mobile robotics enables a more advanced maintenance strategy pursued by infrastructure companies, namely, condition-based maintenance. By providing accurate information about specific sections of a distributed infrastructure, maintenance can be planned before a failure occurs, while the system components are used for the longest possible period of time.

\section{B. Environment negotiation}

An important consideration in implementing an autonomous robotic platform is enabling it to negotiate the environment of the distributed infrastructure. Presently, robots are commonly used for internal inspection of pipes, performing such tasks as leak detection for oil and gas lines, as well as providing a means for visual examination [3-5]. The simple geometry inside of pipes allows these robots to be quite effective. There are numerous examples of different types of drive mechanisms and control algorithms that accomplish this 
task [6-8]. However, implementing robots in a much less structured environment, for example, outside of the pipes, is a major challenge [9]. Although inspection robots usually optimized for specific applications, improvements must be made in the areas of artificial intelligence, mechanical design, power management, and payload capacity for mobile monitoring robots to gain widespread use in distributed infrastructures.

\section{Sensor technologies}

Once the economic justification has been established and problems with delivering the robot to the critical location within the infrastructure are overcome, the ability to detect and locate the parameters of interest must be developed. Adaptation of sensing technologies into the constraints of the robotic platform presents significant scientific challenges, as the issues of size, power management, and the complexity of the diagnostic equipment must be addressed.

The remaining portion of this paper is a further discussion of how these three system-level challenges have been addressed for the mobile monitoring of an underground power distribution system. In this particular example, an autonomous robotic platform traverses along the outside of medium-voltage power cables taking periodic measurements of system parameters.

\section{ECONOMIC ANALYSIS OF MAINTENANCE MethodS}

Today's deregulated power industry relies on many cost-saving methods to stay profitable. While the price of energy can fluctuate wildly, lowering the overall operating cost can provide utilities with significant long-term benefits. Considering only the operating cost, the following equation describes the relative advantages of different maintenance strategies:

$$
\min (C)=\min \left(\lambda_{F} C_{F}+\lambda_{M} C_{M}\right)
$$

where $C$ is the total operational cost, $C_{F}$ is the loss due to failures, $C_{M}$ is the maintenance cost, and $\lambda_{F}$ and $\lambda_{M}$ are the weight factors for $C_{F}$ and $C_{M}$, respectively. Weight factors are typically introduced to accommodate individual judgment calls regarding strategic importance of each component [10]. The variables $C_{F}$ and $C_{M}$ are determined by the maintenance strategies, the aging status of distribution networks, the availability of funding and labor, and other factors.

The occurrence of failures is dependent on maintenance methods. The following observations generally hold true for distributed infrastructures:

1) When no maintenance is performed on a system, failure is unavoidable, and the failure cost reaches its maximum possible value.

2) Increasing maintenance cost can lower the failure cost; the relationship between the two costs is generally nonlinear.

3) When an increased maintenance cost reaches a certain value, it has very small effect on the failure cost.

The numeric values and the relationships between these costs determine the selection of maintenance actions. Three basic levels of maintenance strategies are described in the following sections.

\section{A. Corrective/emergency maintenance (CM)}

Corrective maintenance (CM) means repair or replacement in the system is taken after a failure happens. CM is the easiest and most popular maintenance method since there is no monitoring requirement [11]. Generally, CM is practical only if failure loss and maintenance cost in (1) are small enough. One example of a power network for which $\mathrm{CM}$ is a reasonable choice is a recently installed non-critical distribution network, where the payoff from preventive maintenance is too low. Another example is an underwater cable network, where the costs of routine maintenance are very high with present technologies. If unnecessary monitoring is imposed in these situations, the total operational cost may soar up. Corrective maintenance becomes unacceptable when the right portion of (1) reaches a critical cost level. For example, an unexpected power shutdown of sensitive customers, such as a semiconductor manufacturer, may entail very high cost.

\section{B. Scheduled maintenance (SM)}

Scheduled maintenance (SM), as the name suggests, is planned maintenance carried out in predetermined time intervals to prevent possible failures. SM includes routine monitoring, repair of a network, or part replacement when incipient faults are detected by monitoring or predicted by estimation. It is inherently difficult to determine optimum maintenance time intervals due to the scarcity of real-time information about the system, therefore, the standard used to estimate network components lifetime is generally conservative. As a result, losses are inevitable when network components are replaced prematurely, i.e., $C_{M}$ is increased.

\section{Condition-based maintenance (CBM)}

Condition-based maintenance (CBM) requires information about the actual status of the system. By predicting failures, this strategy helps extending the system's service lifetime and reduces the replacement cost. CBM has received increasing attention in recent years in the power industry as a viable maintenance method for cable networks. A case study of CBM has shown that the performance of a network was increased by $40 \%$ and the maintenance cost was lowered by a factor of seven when cables were repaired instead of being replaced [12]. However, CBM also has disadvantages, for example, the introduced monitoring cost may be very high, or it may be difficult to acquire the accurate status of the system.

\section{Hybrid maintenance}

No single maintenance method is always the most effective for all power systems. For instance, a newly installed network has a low expected failure occurrence, and CM is cost-efficient for the initial period of network life. As time progresses, network components begin to age and the system then requires routine monitoring and maintenance. SM works well for this time period. When a cable system becomes prone to failure and approaches its life expectancy, CBM becomes the best maintenance method. Finally, when repair is no longer effective, replacement of the network is required. Upon replacement of the cable system, the described process, called hybrid maintenance, starts again. The conceptual diagram for 
different maintenance strategies is shown in Fig. 1. As expected, hybrid maintenance provides the minimal running cost, which is presented by the envelope of CM, SM, and CBM.

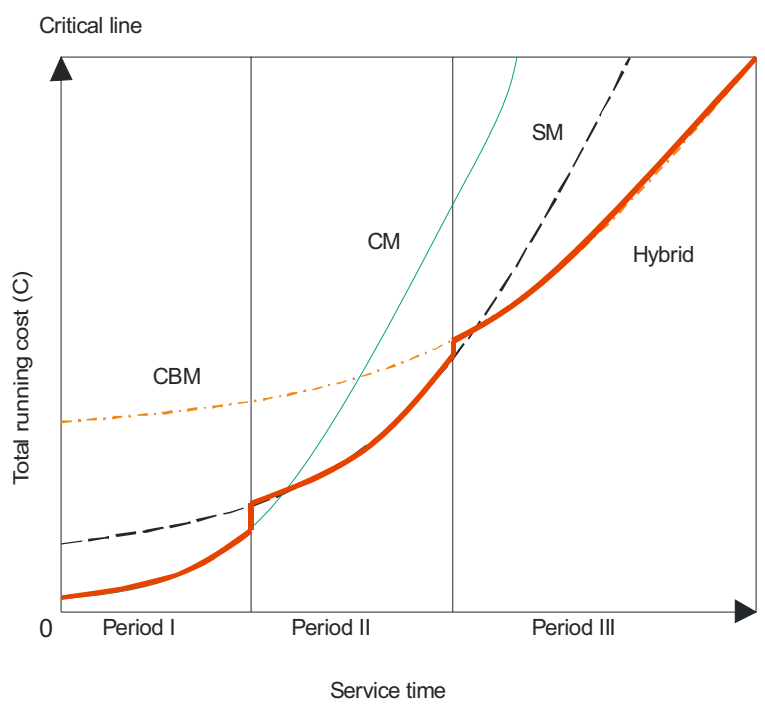

Fig. 1. The total cost trend with the variance of maintenance cost.

\section{THE MoBILE CRAWLING RoBOT}

As already stated, it is crucial for any preventive maintenance technology to obtain accurate information about the system. In this demonstration project, the mobile robot has been implemented to monitor the physical properties of underground cables. The major requirements for the platform are: the ability to operate over large distances, operate within the physical constraints of the cable environment, negotiate obstacles within the environment, and carry the instruments needed for cable inspection. A detailed description of the platform design can be found in [13].

\section{A. Mechanical design}

The underground cable environment is not as geometrically simple as a pipe and requires a much more adaptable design. To negotiate effectively the unstructured environment of a power distribution conduit, a multi-segmented robot design has been implemented. This design was selected because it allows enhanced turning ability, stability in turning, subsections that are interchangeable, and the ability to support larger payloads.

The present platform has two segments, one devoted to robot control and data processing, and the other devoted to sensor payloads. This design allows flexibility in interchanging subsections for different service and diagnostic tasks. In order to keep the robot on the cable, each individual segment of the robot base is equipped with a set of stabilizers. These stabilizers are able to retract so that the robot may better negotiate obstacles, such as a splice in the cable or a mounting bracket.

While many of the sensors do not require contact with the cable, some, such as the acoustic emission sensor, must have proper contact with the cable surface in order to take accurate measurements. A servo-powered linear actuator is used to periodically lower sensing equipment to monitor the power distribution line. Fig. 2 shows the robot on a cable and trough installation in an underground service tunnel. In the foreground is the front drive section, followed by a set of stabilizers and a section for carrying sensors.

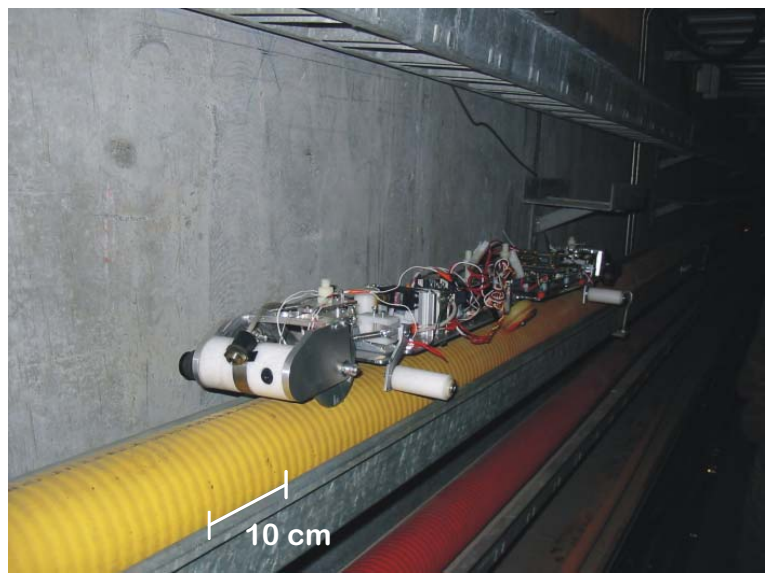

Fig. 2. Photograph of the robotic platform for the mobile monitoring of underground power cables.

\section{B. Overview of control system}

The control system for the robot includes an autonomous mode, which performs the following functions: data acquisition and processing, navigation, and robot control. To accomplish this, a multiprocessor architecture has been implemented for the main control board so that tasks can be done simultaneously. When in signal range, a host computer can communicate with the robot using $915 \mathrm{MHz}$ wireless communication modules. At that time, the operator can aid the robot in obstacle negotiation, give new instructions and global commands, and remotely view sensor output. While this method requires distances less then one hundred meters and a line of sight between the host and the robot, the autonomous functions of the robot makes long-range communications unnecessary.

\section{Monitoring strategies}

The robot can perform monitoring tasks either autonomously or under control of a remote human operator. When running autonomously, the robot will travel along the cable, stopping periodically to take measurements. If this general scan routine detects a possible failure, the robot will repeat the scan of the area, taking detailed measurements to verify and locate the failure. After locating a problem, the robot will either to transmit this information back to the host computer or will save the relevant sensor data to the memory on the control board. This data can later be recalled and analyzed when the robot comes within range of the host computer. If necessary, the robot can be sent back to the damaged section of cable to perform additional scans. Fig. 3 shows the control strategies, which are implemented on this robotic platform. 


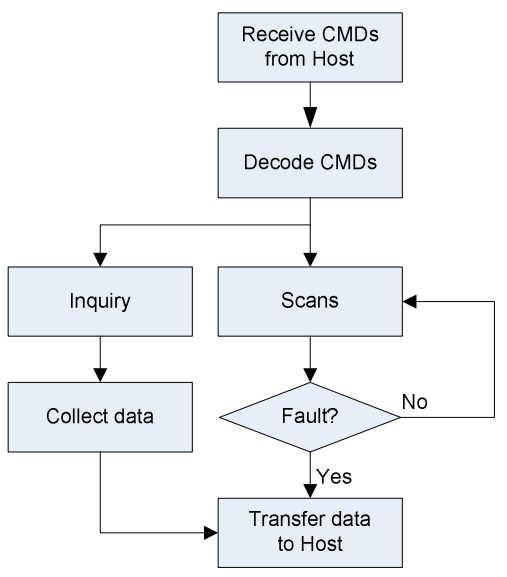

Fig. 3. Routine operation algorithm of the robot (CMD stands for command).

\section{Sensor Selection, Design, And Testing}

The selection and integration of sensors is challenging because the following criteria must be met: a) sensors must be able to measure physical variables that help the evaluation of cable status; b) sensors must be small and lightweight, thus allowing them to be easily incorporated into the robot platform; c) sensors must be sufficiently rugged to withstand mechanical and environmental impact.

Four types of diagnostic sensors were chosen for this robot: an infrared sensor for detection of hot spots, a fringing electric field (FEF) sensor for detection of water trees, an acoustic sensor for detection of partial discharges, and a video sensor for inspection of mechanical cracks. The infrared sensor and the video sensor can work continuously when the robot patrols the cable. The acoustic and the FEF sensor can be used to estimate the health status of cables when sensors have a proper contact with the cable (which requires full stop of the robot.) The FEF sensor and acoustic sensor may also collect useful data when the robot is moving, although with lesser accuracy. Fig. 4 illustrates these operating modes. The quantity of data collected by each sensor is proportional to the height of the shaded areas.

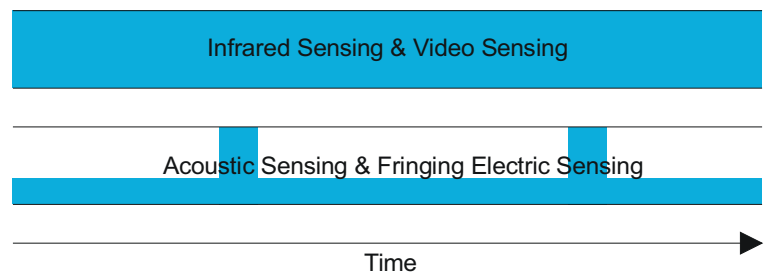

Fig. 4. The operating modes of sensors.

The following subsections provide a discussion on sensor selection and present representative experimental data.

\section{A. Infrared sensors for hot spot detection}

Excessive heat build-up significantly contributes to the premature breakdown of the insulation in distribution cable networks. Factors that cause overheating include current overloading, physical damage, insulation aging, partial discharges, changes in ambient temperature, and proximity to other cables and water/steam pipes [14]. Many of these overheating problems result in hot spots, since overheating rarely affects the entire length of the cable.

Infrared sensors do not need physical contact with the cable. Therefore, they can continuously take temperature readings as the robot travels along the cable. Fig. 5 shows an artificially created hot spot measured by the Raytek ${ }^{\circledR}$ MID infrared sensor in laboratory conditions. The measurement was taken in an autonomous mode while the robot was traversing along the cable. The trigger condition in this case was the $15^{\circ} \mathrm{C}$ increase above the mean ambient temperature. As experiments continue, much more sophisticated pattern recognition algorithms will be used for pre-processing of sensor data.

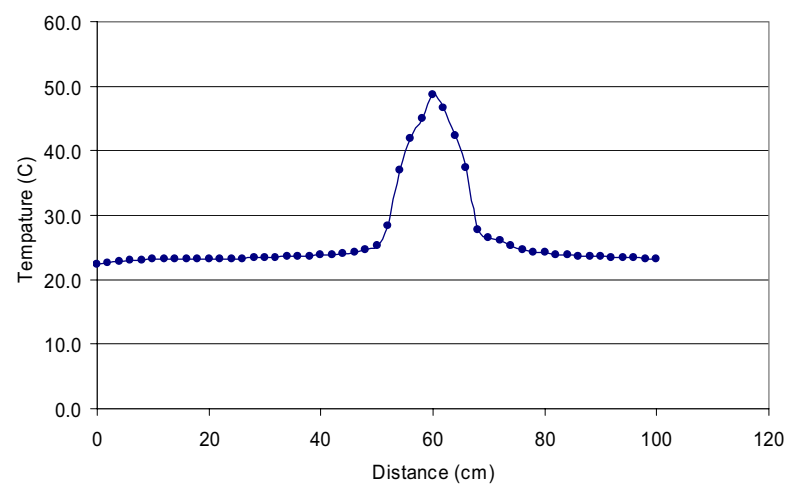

Fig. 5. Infrared sensor measurement of a hot spot taken as the robot traversed along a cable.

\section{B. Sensors for partial discharges}

Partial discharges (PDs) occur when a failure in the cable insulation causes a rapid energy release, usually resulting in a detectable acoustic vibration. In many cases, PDs are a precursor to total cable failure, and serve as an important indicator for estimating the remaining service life of cables.

Acoustic sensing has certain advantages over other methods of partial discharge detection. It is free from electrical-interference, easy to implement for a broad range of frequencies, and allows high data acquisition rates. For these reasons, acoustic sensors are typically used for monitoring of partial discharge activity in transformers and switchgear. However, the attenuation of the acoustic waves over distance makes it impractical to use acoustic emission sensors as stationary distributed nodes in cable networks. The ability of the mobile robotic platform to transport the sensors along the cable dramatically increases the usefulness of acoustic sensors for diagnostics of electric power cables.

Fig. 6 shows the time domain and frequency signal of PDs captured in two different situations: (a) the sensor is close to the PD source (several centimeters); (b) the sensor is far from the PD source (several meters). The acoustic emission sensor R6I from Physical Acoustics Corporation is used in both cases. In the first case, the acoustic signal's amplitude is very high and it contains many high frequency components. In the second case, the amplitude of the acoustic signal is much smaller, and the major frequency components are in low frequency bands. Intuitively, it is a valid result, as high frequencies attenuate faster than low frequencies. These measurements demonstrate the ability to locate the PD region by processing acquired data 
both in time and in frequency domain.
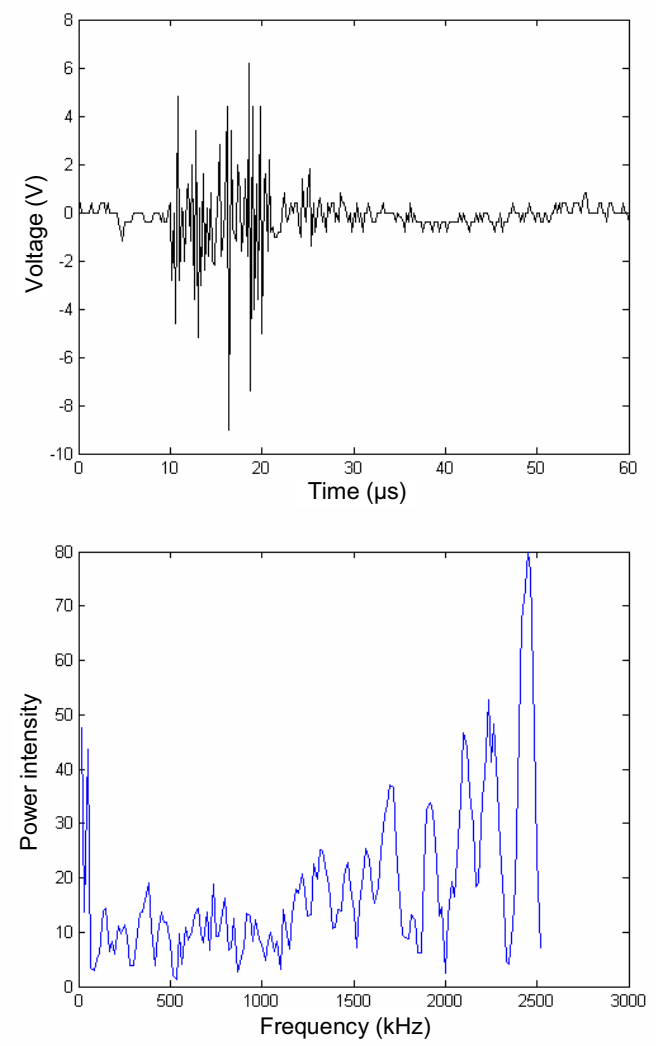

Fig. 6. (a) PDs captured when the PDs source and the sensor are close $(20 \mathrm{~cm})$.
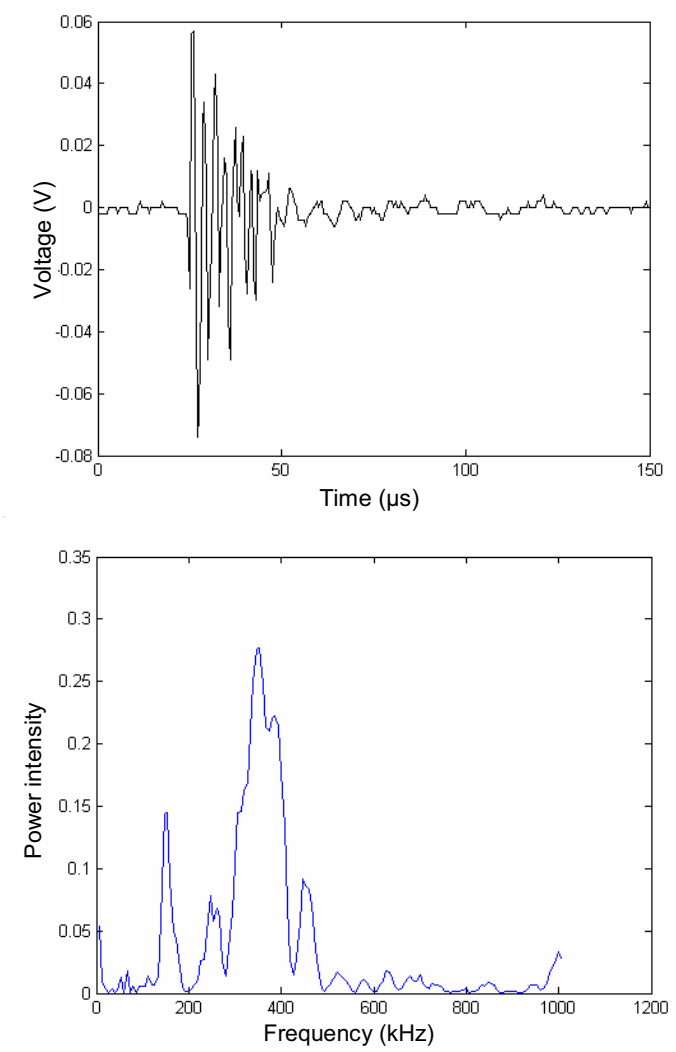

Fig. 6. (b) PD data acquired when the PD source and the sensor are farther away from each other $(5 \mathrm{~m})$.

\section{Dielectrometry sensors}

Measurement of the dielectric properties of the cable insulation can provide a powerful diagnostics alternative when compared to the capabilities of the acoustic emission detector. For example, a partial discharge event may be intermittent due to environmental conditions. A cable with a small crack may remain dry and in working condition during a hot summer. As the weather changes and the cable becomes wet, the water in the crack may act as a conduit for electrical discharges. In this case, the acoustic sensor would not detect the partial discharge resulting form the water tree, unless the robot was in the right place at the right time.

A fringing electric field dielectric spectroscopy sensor can test the properties of insulating material rather then the resulting effects. In order to locate water trees, the sensor is looking for the presence of water. In the presented robotic platform design, the sensor head is periodically lowered from the robot platform onto linear actuators to inspect the distribution cable.

At this stage, only proof-of-concept measurements have been conducted with simple experimental setups. In such an experiment, measurements of water uptake in the XLPE cables were conducted with a fringing electric field sensor and the sensitivity to the presence of water was demonstrated. Detailed experiments and data analysis are subject of future work.

Video cameraFig. 7 shows an image captured from the onboard video camera, a simple and useful additional tool for the cable inspection. Coupled with a wireless user interface, an operator viewing real-time video can perform a visual inspection of the cable that may not be safely or easily accessed by maintenance personnel, for example, in a nuclear power plant environment. The operator can also aid in advanced obstacle avoidance and remotely troubleshoot problems, thereby enhancing the capabilities of the robot.

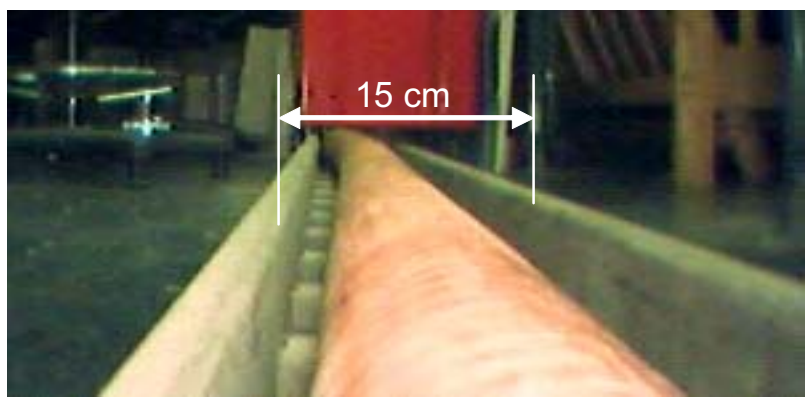

Fig. 7. A view from the on-board camera transmitted wirelessly to the host computer.

Although these sensors are specially selected for the underground high-voltage cables, they represent the validity of sensing technologies in the generic distributed infrastructure.

\section{FUTURE WORK}

Also currently in progress, is the enhancement of the mobile monitoring approach with a ubiquitous Radio-Frequency Identification (RFID) [15] enhanced sensor network. Energy-scavenging RFID tag can be placed by the robots at critical locations and later used either by maintenance staff or detected by mobile robots in future runs. In this way, the 
problem of losing the accuracy of the location due to accumulated errors in the shaft encoders can be addressed. Furthermore, by integrating RFID and the simple threshold sensors, the sensor network can become heterogeneous, including a mobile agents as well as stationary nodes. Fig. 8 shows the enhanced RFID tag configuration developed for this task. The history data can be recorded and transferred wirelessly to the robot when it travels to the RFID tag location. This information could greatly enhance the diagnostic ability of the mobile robot.

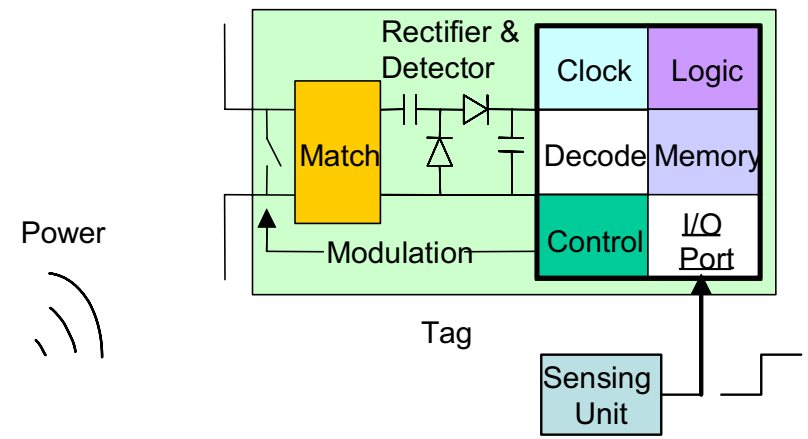

Fig. 8. The RFID-enhanced sensor node.

\section{CONCLUSIONS}

A mobile autonomous robot for detection of incipient faults in distributed infrastructure has been developed and tested in laboratory and in field conditions. Mechanical design and autonomous control system of the robot are optimized for the requirements of the operating environment, in this case, underground tunnels of distribution power systems. The analysis of detectable physical phenomena led to a selection of necessary sensing principles. Four types of sensors for measurement of physical properties of the distributed infrastructure were integrated into the mobile platform and tested in laboratory conditions. The presented mobile inspection method provides a viable solution to the task of monitoring and maintaining underground distributed infrastructure.

\section{ACKNOWLEDGEMENTS}

The authors would like to express their gratitude to Ryan Wistort for assistance with designing, assembling, and operating experimental setups. The research projects of the undergraduate students were supported by the University of Washington Mary Gates Foundation and the Electric Energy Industrial Consortium. The authors also gratefully acknowledge the invaluable assistance of Mark Kirschenbaum of the University of Washington Power Plant with experiments.

\section{REFERENCES}

[1] E. David, N. Amyot, and J.-F. Drapeau, "Diagnostic of Field Aged Cables and Accessories by Time-Domain Dielectric Spectroscopy," 2003 Annual Report on Conference on Electrical Insulation and Dielectric Phenomena, 2003, pp. 165-170.

[2] N. H. Ahmed and N. N. Srinivas, "On-Line Partial Discharge Detection in Cables," IEEE Transactions on Dielectrics and Electrical Insulation, vol. 5, no. 2, pp. 181-188, 1998.
[3] A. Zagler and F. Pfeiffer, "'MORITZ" a Pipe Crawler for Tube Junctions," Proceedings of IEEE International Conference on Robotics and Automation, vol. 3, 2003, pp. 2954-2959.

[4] S. G. Roh, S. M. Ryew, J. H. Yang, and H. R. Choi, "Actively Steerable in-Pipe Inspection Robots for Underground Urban Gas Pipelines," Proceedings 2001 ICRA IEEE International Conference on Robotics and Automation, vol. 1, 2001, pp. 761-766.

[5] H. T. Roman, B. A. Pellegrino, and W. R. Sigrist, "Pipe Crawling Inspection Robots: an Overview," IEEE Transactions on Energy Conversion, vol. 8, no. 3, pp. 576-583, 1993.

[6] A. M. Bertetto and M. Ruggiu, "In-Pipe Inch-Worm Pneumatic Flexible Robot," Proceedings of 2001 IEEE/ASME International Conference on Advanced Intelligent Mechatronics, vol. 2, 2001, pp. 1226-1231.

[7] J. A. Galvez, P. Gonzalez de Santos, and F. Pfeiffer, "Intrinsic Tactile Sensing for the Optimization of Force Distribution in a Pipe Crawling Robot," IEEE/ASME Transactions on Mechtronics, vol. 6, no. 1, pp. 26-35, 2001.

[8] S. Guo, Y. Sasaki, and T. Fukuda, "A New Kind of Microrobot in Pipe Using Driving Fin," Proceedings of 2003 IEEE/ASME International Conference on Advanced Intelligent Mechatronics, vol. 2, 2003, pp. 697-702.

[9] B. Jiang and A. V. Mamishev, "Robotic Monitoring of Power Systems," IEEE Transactions on Power Delivery, vol. 19, no. 3, pp. 912-918, July 2004.

[10] Y. Cheng, T. S. Chung, C. W. Yu, C. Y. Chung, M. Zeng, and X. Sun, "Application of Reliability-Centered Stochastic Approach and FMECA to Conditional Maintenance of Electric Power Plants in China," Proceedings of the 2004 IEEE International Conference on Electric Utility Deregulation, Restructuring and Power Technologies, vol. 2, 2004, pp. 463-467.

[11] J. H. Williams, A. Davies, and P. R. Drake, Condition-based maintenance and machine diagnostics, Chapman \& Hall, 1994.

[12] W. Reder and D. Flaten, "Reliability Centered Maintenance for Distribution Underground Systems," IEEE Power Engineering Society Summer Meeting, vol. 1, 2000, pp. 551-556.

[13] B. Jiang, A. P. Sample, R. Wistort, and A. V. Mamishev, "Autonomous Robotic Monitoring of Underground Cable Systems," Proceedings of International Conference on Advanced Robotics, 2005.

[14] L. Lamarre, D. Fournier, and R. Morin, "Early Detection of Faults in Underground Distribution Cable Joints by Partial Discharge Measurements," Proceedings of the 4th International Conference on Properties and Applications of Dielectric Materials, 1994, pp. 864-867.

[15] K. Finkenzeller, RFID handbook: fundamentals and applications in contactless smart cards and identification, 2 ed., Wiley, 2003. 